\title{
DEFICIENT PROTEIN C AND PROTEIN S INDUCED ACUTE VENOUS MESENTERIC ISCHEMIA: A CASE REPORT
}

\author{
Darwin Britto Devadhason ${ }^{1}$, Alankrith Ramesh Kashyap², Mohamed Zakariya ${ }^{3}$, Mohamed Musthafa $^{4}$, Arun Prasanth ${ }^{5}$ \\ ${ }^{1}$ Assistant Professor, Department of General Surgery, SRM Medical College, Hospital and Research Centre, Chennai. \\ ${ }^{2}$ Post Graduate, Department of General Surgery, SRM Medical College, Hospital and Research Centre, Chennai. \\ ${ }^{3}$ Post Graduate, Department of General Surgery, SRM Medical College, Hospital and Research Centre, Chennai. \\ ${ }^{4}$ Professor, Department of General Surgery, SRM Medical College, Hospital and Research Centre, Chennai. \\ 5 Post Graduate, Department of General Surgery, SRM Medical College, Hospital and Research Centre, Chennai.
}

\section{ABSTRACT}

\section{BACKGROUND}

A 35 year old lady presented with unresolved severe abdominal pain and vomiting. She was diagnosed to have superior mesenteric vein thrombosis with gangrenous small bowel and multiple splenic infarcts secondary to Protein $\mathrm{C}$ and Protein $\mathrm{S}$ deficiency. She underwent emergency explorative laparotomy and extensive small bowel resection and anastomosis and splenectomy. This is to stress the importance of keeping mesenteric ischemia as an important differential diagnosis in cases of acute abdomen.

\section{KEYWORDS}

Venous Mesenteric Ischemia, SMV Thrombosis, Protein C, S Deficiency.

HOW TO CITE THIS ARTICLE: Devadhason DB, Kashyap AR, Zakariya M, et al. Deficient protein c and protein s induced acute venous mesenteric ischemia: a case report. J. Evolution Med. Dent. Sci. 2016;5(37):2250-2253, DOI: 10.14260/jemds/2016/522

\section{INTRODUCTION}

Mesenteric Ischemia is an unexpected cryptogram that accounts for $2 \%$ of all gastrointestinal emergencies and can result in a mortality rate of $50 \%$ to $90 \%$ despite intensive care; hence prompt recognition of symptoms and treatment is essential for patient survival.[1] Though rare, the main predicament with mesenteric ischemia is that intestinal gangrene almost always sets in by the time it is recognized, thus posing a challenge to the surgeon.[2] About 5-10\% of mesenteric ischemia is accredited to Acute Venous Mesenteric Ischemia (AVMI), impaired intestinal venous drainage due to Superior Mesenteric Vein (SMV) occlusion; causing vascular engorgement, swelling and hemorrhage in the bowel wall.[1] SMV occlusion can be either primary (Idiopathic) or secondary to diverse conditions, one of them being secondary to Protein $\mathrm{C}$ and Protein S deficiency. ${ }^{[3]}$ Protein $\mathrm{C}$ and S deficiency are infrequent having an autosomal dominant pattern of inheritance, with a prevalence rate of $0.2-0.3 \%$ and $0.2-0.5 \%$ respectively.[4,5] Protein $\mathrm{C}$ and Protein $\mathrm{S}$ are natural anticoagulants and their deficiency can lead to venous thrombosis.[4] The average age of Mesenteric Vein Thrombosis (MVT) presentation ranges from 45 to 60 years with males being slightly more affected than females.[6]

We report a rare case of Acute Venous Mesenteric Ischemia caused by SMV thrombosis as a result of Protein C, Protein S deficiency.

\section{CASE REPORT}

A 35 year old lady presented with complaints of abdominal pain for 10 days, which increased in severity since in 1 day.

Financial or Other, Competing Interest: None.

Submission 26-03-2016, Peer Review 20-04-2016,

Acceptance 26-04-2016, Published 09-05-2016.

Corresponding Author:

Dr. Alankrith Ramesh Kashyap,

Room 303, PG Medical Gents Hostel,

SRM College Hospital \& Research Centre,

SRM Nagar, Kattankulathur,

Chennai-603203.

E-mail: koolchant@gmail.com

DOI: 10.14260/jemds/2016/522
The pain started in the upper abdomen 10 days back and is present all over the abdomen. Since 1 day she has developed abdominal distension accompanied with vomiting almost 10 episodes, containing food particles. The patient was not a diabetic, hypertensive, heart disease, deep vein thrombosis or smoker/alcoholic. No history of Oral Contraceptive (OCPs) use or previous surgeries.

On examination: the patient was conscious and oriented, showed signs of dehydration.

Pulse $-110 /$ minute.

Blood Pressure 100/70 mm Hg.

Per abdomen showed generalized distension, with diffuse tenderness along with guarding and rigidity. Bowel Sounds-absent.

Per rectal examination revealed feces, no malena or hematochezia. Her other systems were normal. On four quadrant tapping reddish brown colored fluid was aspirated.

A clinical diagnosis of acute hemorrhagic pancreatitis or perforative peritonitis was made. Her Ultrasound abdomen/pelvis: body and tail of pancreas appears bulky with ascites X-ray Abdomen erect: ground glass appearance.

Chest X-ray: Normal Contrast Enhanced CT(CECT) whole abdomen: SMV thrombosis with splenic vein thrombosis extending into portal vein multiple splenic infarcts, bowel wall edema with ascites (Figure 1, 2, 3, 4)

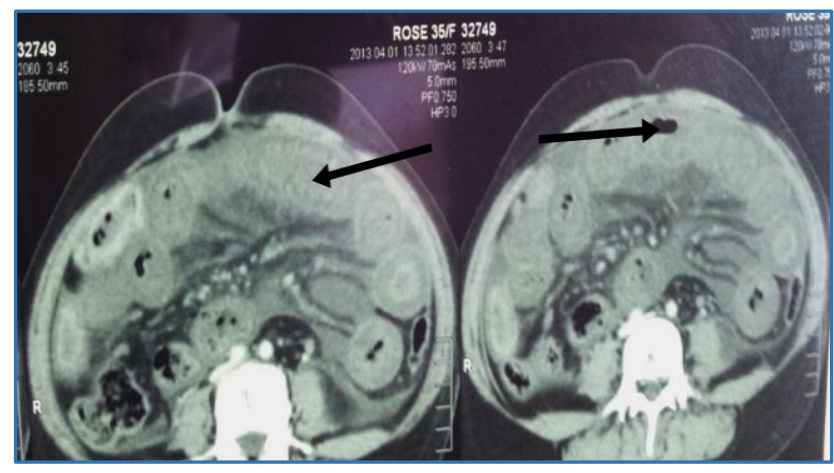

Fig. 1: Bowel Wall Thickening and Edema 


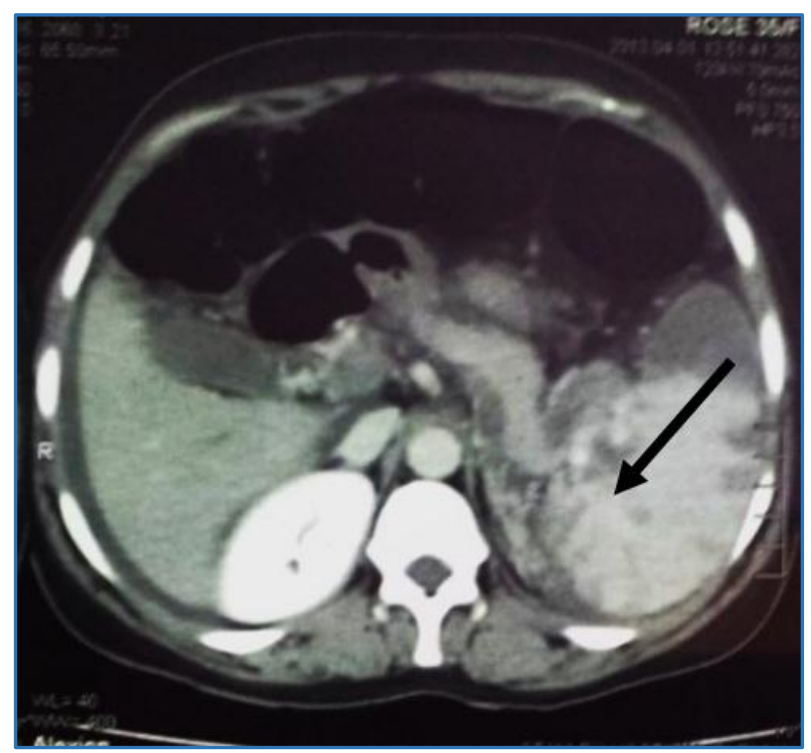

Fig. 2: Multiple Splenic Infarcts

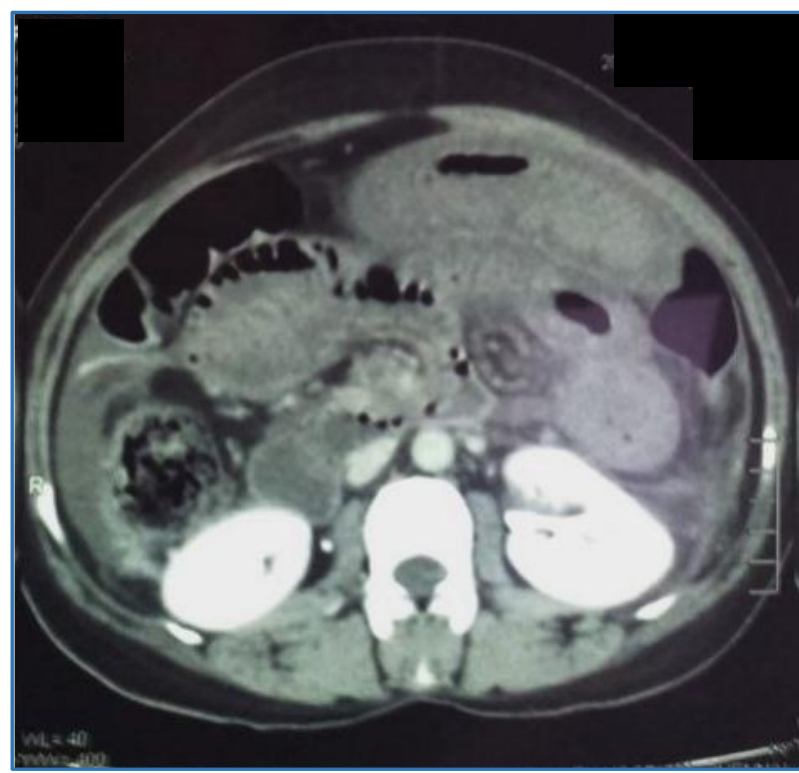

Fig. 3

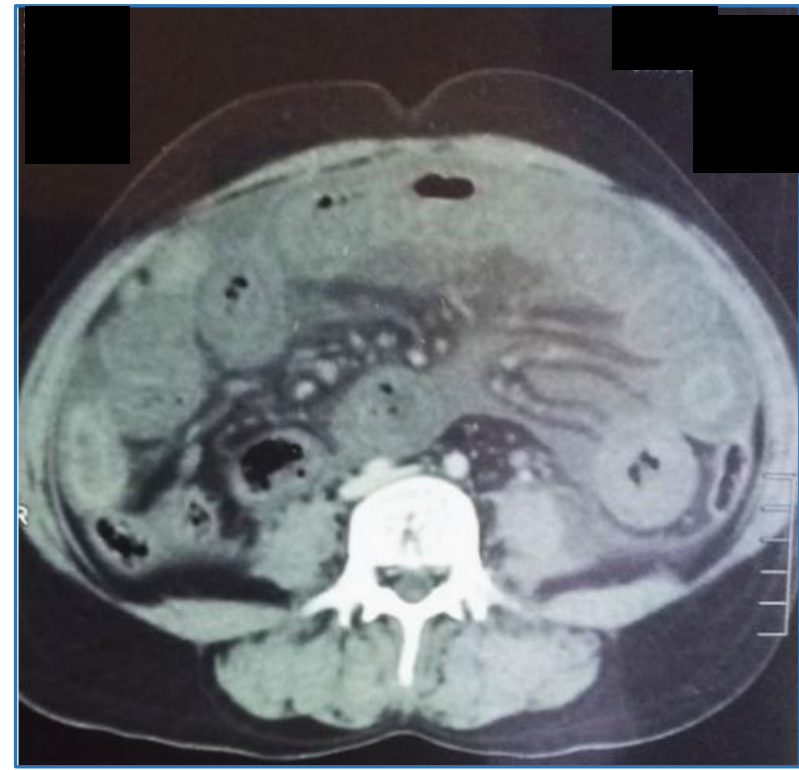

Fig. 4
Along with blood investigations, coagulation profile, haemoglobinopathy and immunology screening was sent and planned for emergency explorative laparotomy.

\section{BLOOD INVESTIGATIONS}

\section{Hemoglobin}

$10 \mathrm{~g} \%$ (Normal: $12-14 \mathrm{~g} \%$ ), Total count: 21000 cells/cu. mm (Normal: 4000-11,000 cells/cu.mm), Platelets: 4.2 lakh/cu. mm (1.5-4.5 lakh cells/cu.mm), Blood Urea: $45 \mathrm{mg} / \mathrm{dl}$ (Normal 15-48 mg/dl), Serum creatine: $1 \mathrm{mg} / \mathrm{dl}$ (0.5-1.5 mg/dl).

\section{Coagulation Profile}

PT : 11 sec.

INR : 0.8 .

Protein s activity: 19\% (Normal: 72 to $116 \%$ ).

Protein c activity: $32 \%$ (Normal: 70 to $130 \%$ ).

Antithrombin : 78\% (Normal : 80 to 120\%).

\section{Hemoglobinopathy Screening}

fraction $\mathrm{Hb} \mathrm{F}: 0.9 \%$ Normal in adults : $<2 \%$.

Hb A0: $84.9 \%$ normal: $85 \%$ - 95\%.

Hb A2: 2.5\% normal: $2.0-3.7 \%$.

\section{Immunology (Blood)}

ds - DNA Antibody - Negative.

U1 nRNP Antibody - Negative.

Cardiolipin Antibody -Negative.

Phospholipd Antibody- Negative.

\section{Electrocardiogram: Normal.}

\section{Intraoperative Findings}

SMV with Splenic vein thrombosis with multiple splenic infarcts. Gangrene of small intestine from $15 \mathrm{~cm}$ distal to Duodeno-jejunal (DJ) flexure to $15 \mathrm{~cm}$ proximal of ileo-caecal junction. 1 litre of reddish brown peritoneal fluid drained (Figure 5, 6, 7, 8).

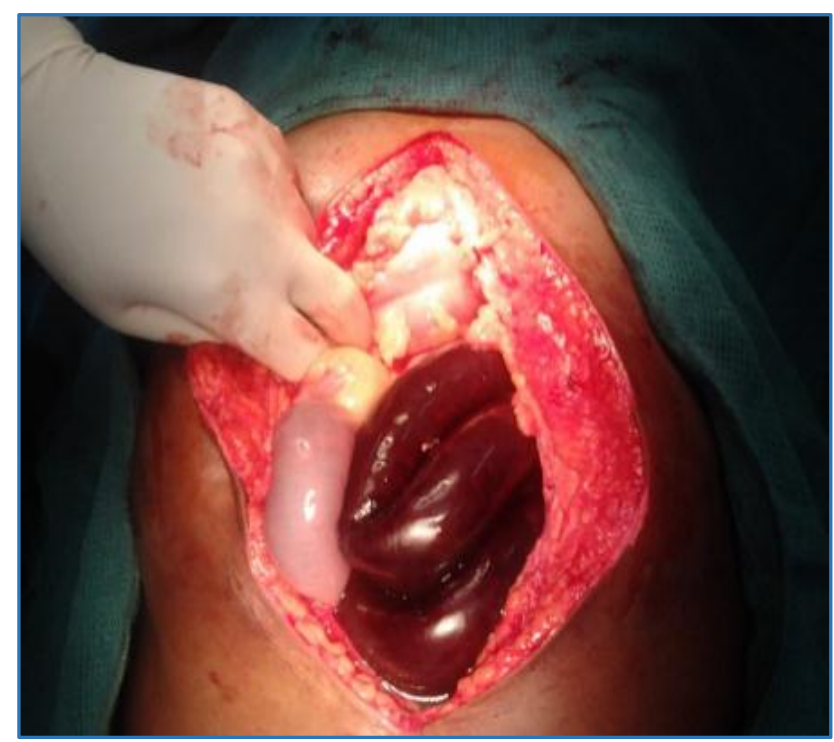

Fig. 5: Gangrenous Small Bowel 


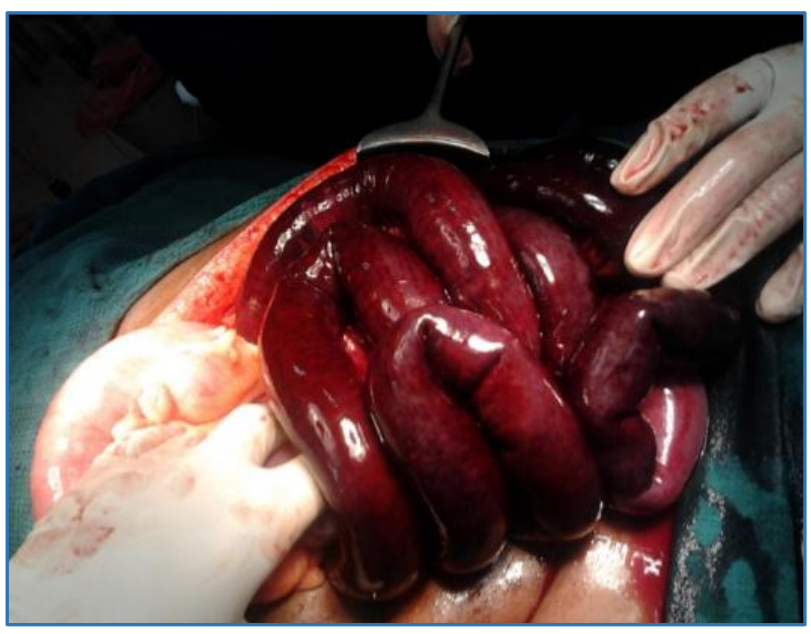

Fig. 6: Gangrenous Small Bowel

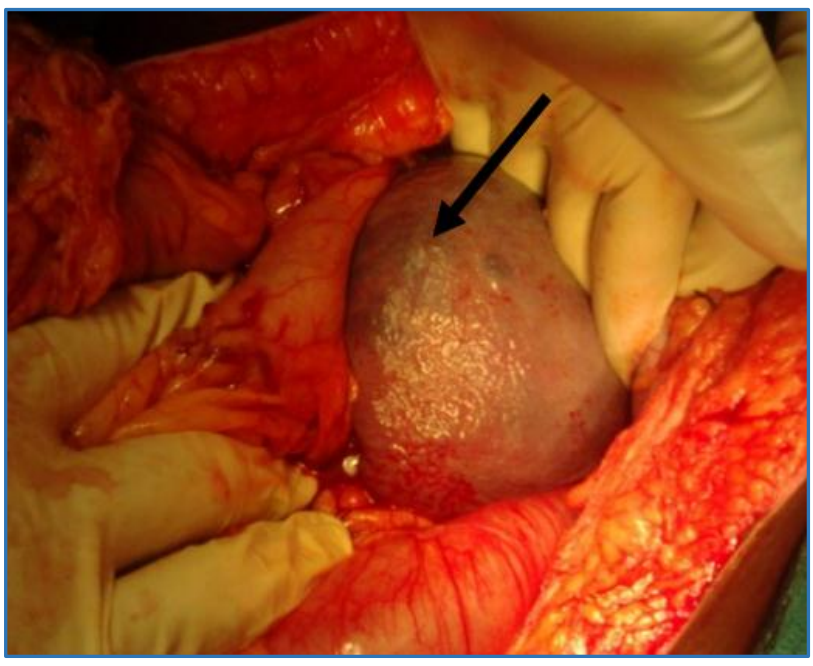

Fig. 7: Multiple Splenic Infarcts

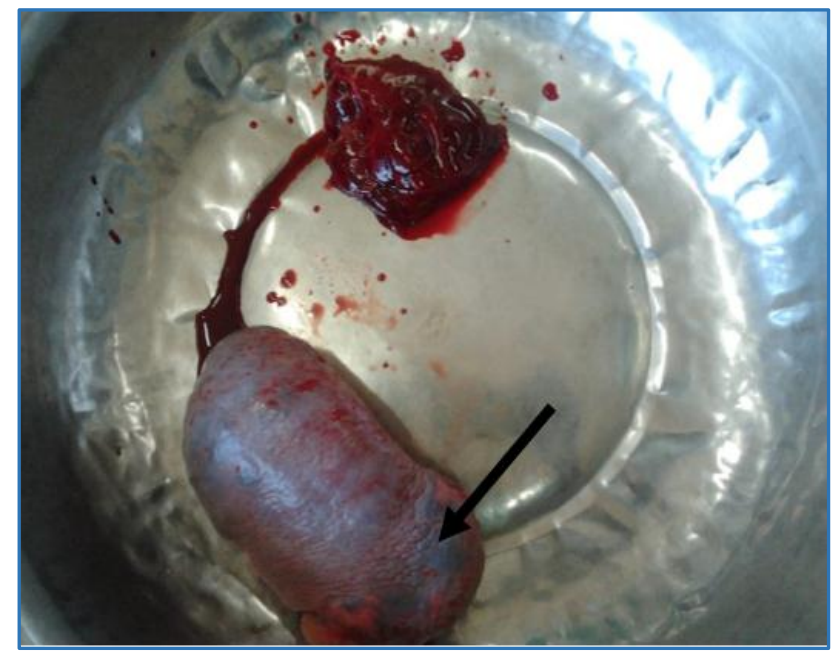

Fig. 8: Splenectomy Specimen

Patient underwent explorative laparotomy with splenectomy with resection of gangrenous bowel segment followed by jejuno-ileal anastomosis and peritoneal lavage.

Post-operatively patient was started on Low Molecular weight heparin. After 10th post-operative period patient was switched over to oral anticoagulant and dose was adjusted to maintain INR within 2-3.

\section{DISCUSSION}

AVMI contributes to $15-40 \%$ mortality rate mainly due to the delay in diagnosis of acute MVT which mysteriously presents with very non-specific clinical features despite at an advanced stage; nevertheless it still carries a relatively better prognosis than other forms of acute mesenteric ischemia, if caught in time.[6,7] Acute MVT has bizarre symptoms, only hallmark of this malady being abdominal pain out of proportion to the clinical signs, with peritoneal signs indicating bowel infarction has already set in.[6,7] The involved territory in acute MVT is ileum (64-83\%), jejunum (50-81\%) followed by colon and duodenum with $14 \%$ and $4 \%$ respectively.

Acute MVT is abdominal pain less than 4 weeks in duration and severity is based on the location, extent and timing of thrombus formation within the SMV, with many patients seeking medical attention only after 5-14 days of continuous unresolved abdominal pain.[6] Delayed presentation may include marked leukocytosis, dehydration, fever, acidosis, signs of peritonitis and thus becomes a surgical emergency.[8] Predisposing condition for MVT include hypercoaguable states like Factor $\mathrm{V}$ mutation, Antithrombin III, Protein C,S deficiency, vasculitis, malignancy, oral contraceptives, Cirrhosis and portal hypertension, acute pancreatitis or any previous surgery.[3]

Hypercoaguable states like Protein C, Protein S deficiency leads to thrombosis beginning in intramural venules, vasa recta and venous arcades without any scope for collateral drainage development, thus causing immediate bowel infarction. [6] Contrast Enhanced Computed Tomography (CECT) abdomen can swiftly reveal the diagnosis with characteristic finding being: hypodensity in trunk of SMV (indicating thrombus), thickened small bowel segment, intraperitoneal fluid collection. ${ }^{2]}$ Conservative management of MVT is not applicable when CECT Abdomen shows bowel infarction with peritoneal signs set in and patient shows signs of clinical deterioration, resection of the gangrenous segment with or without anastomosis and immediate intraoperative/post-operative hepariniztion is the treatment of choice. ${ }^{[6,7]}$

\section{CONCLUSION}

When a patient comes with vague abdominal pain out of magnitude to the clinical findings, with no definitive ascribable cause; mesenteric ischemia should be one of the crucial differential diagnosis. Liberal use of CECT abdomen to evaluate such cases of conundrum can promptly diagnose Acute Mesenteric Venous Thrombosis, which relatively carries a better prognosis. Heparinization with or without surgery according to the patient's status and investigations to establish an identifiable cause can genuinely benefit and promote patient survival.

\section{REFERENCES}

1. Reginelli A, Lacobellis F, Berritto D, et al. Mesenteric ischemia: the importance of differential diagnosis for the surgeon. BMC Surgery 2013;13(2):S51.

2. Schein Moshe, Rogers Paul N. Schein common sense emergency abdominal surgery. Springer-Verlag Berlin Heidelberg 2005;2nd edn:197-204.

3. Ahmed N Assar. Acute mesenteric ischaemia: facts and perspectives. British Journal of Hospital Medicine 2008;69(12):634-640. 
4. Caspers M, Pavlova A, Driesen J, et al. Deficiencies of antithrombin, protein $\mathrm{C}$ and protein S - Practical experience in genetic analysis of a large patient cohort. Thromb Haemost 2012;108(2):247-257.

5. Wypasek Ewa, Undas Anetta. Protein $C$ and protein $S$ deficiency- practical diagnostic issues. Adv Clin Exp Med 2013;22(4):459-467.

6. Harnik Ian G, Brand Lawrence J. Mesenteric venous thrombosis. Vascular Medicine 2010;15(5):407-418.
7. Moh'd Al Salamah Saleh, Mirza Shaukat Mahmood. Acute mesenteric venous thrombosis: management controversies. JK Practitioner 2004;11(4):242-247.

8. Townsend CM. Sabiston textbook of surgery. The biological basis of modern surgical practice. Philadelphia: Saunders 2012;19th edn:1177. 